\title{
Retinoid X Receptor $\alpha$ Overexpression Alleviates Mitochondrial Dysfunction-induced Insulin Resistance through Transcriptional Regulation of Insulin Receptor Substrate 1
}

\author{
Seung Eun Lee,, Young Do Koo ${ }^{1,2}$, Ji Seon Lee', Soo Heon Kwak', Hye Seung Jung', Young Min Cho', \\ Young Joo Park', Sung Soo Chung, ${ }^{1, *}$ and Kyong Soo Park ${ }^{1, *}$
}

\begin{abstract}
Mitochondrial dysfunction is associated with insulin resistance and diabetes. We previously showed that retinoid $X$ receptor $\alpha(R X R \alpha)$ played an important role in transcriptional regulation of oxidative phosphorylation (OXPHOS) genes in cells with mitochondrial dysfunction caused by mitochondrial DNA mutation. In this study, we investigated whether mitochondrial dysfunction induced by incubation with OXPHOS inhibitors affects insulin receptor substrate 1 (IRS1) mRNA and protein levels and whether RXR $\alpha$ activation or overexpression can restore IRS1 expression. Both IRS1 and RXR $\alpha$ protein levels were significantly reduced when C2C12 myotubes were treated with the OXPHOS complex inhibitors, rotenone and antimycin $A$. The addition of RXR $\alpha$ agonists, 9-cis retinoic acid (9cRA) and LG1506, increased IRS1 transcription and protein levels and restored mitochondrial function, which ultimately improved insulin signaling. $R X R \alpha$ overexpression also increased IRS1 transcription and mitochondrial function. Because RXR $\alpha$ overexpression, knock-down, or activation by LG1506 regulated IRS1 transcription mostly independently of mitochondrial function, it is likely that $R X R \alpha$ directly regulates IRS1 transcription. Consistent with the hypothesis, we showed that RXR $\alpha$ bound to the IRS1 promoter as a heterodimer with peroxisome proliferatoractivated receptor $\delta$ (PPAR $\delta$ ). These results suggest that $R X R \alpha$ overexpression or activation alleviates insulin resistance by increasing IRS1 expression.
\end{abstract}

\footnotetext{
${ }^{1}$ Department of Internal Medicine, Seoul National University College of Medicine, Seoul 110-744, Korea, ${ }^{2}$ These authors contributed equally to this work.

*Correspondence: kspark@snu.ac.kr (KSP); suschung@snu.ac.kr (SSC)

Received 17 October, 2014; revised 22 December, 2014; accepted 22 December, 2014; published online 25 February, 2015
}

Keywords: insulin receptor substrate 1, insulin resistance, itochondrial dysfunction, retinoid $\mathrm{X}$ receptor $\alpha$

\section{INTRODUCTION}

It is well known that insulin resistance precedes the development of diabetes. There are many reports suggesting that impaired mitochondrial function is associated with insulin resistance and diabetes. Previous studies found that the mitochondrial oxidative capacity for glucose and lipids is reduced in the skeletal muscle of type 2 diabetes patients (Kelley and Simoneau, 1994; Lowell and Shulman, 2005; Simoneau and Kelley, 1997). In addition, mitochondrial content also is reduced in the muscle of obese and diabetes patients (Kelley et al., 2002; Ritov et al., 2005). There are several theories to explain the association between mitochondrial dysfunction and insulin resistance. For example, specific lipid metabolites (e.g., ceramide and diacylglycerol) accumulate when mitochondrial function is impaired, and they activate protein kinase $\mathrm{C}$, which inhibits insulin signaling through serine/threonine phosphorylation of insulin receptor substrate (IRS) (Morino et al., 2005). A recent study reported that reactive oxygen species (ROS) production is increased in the mitochondria of obese and insulinresistant animal models or humans (Anderson et al., 2009). Although the precise mechanism has not been elucidated, higher ROS levels promote serine/threonine stress kinase activity, which inhibits the insulin signaling pathway (FisherWellman and Neufer, 2012). Even though these observations provide evidence for an association between mitochondrial dysfunction and insulin resistance, whether mitochondrial dysfunction is required for the development of insulin resistance is not known (Martin and McGee, 2014).

IRS is an important regulator of insulin signaling, and its activation is inhibited by phosphorylation at serine residues. Inflammatory cytokines such as tumor necrosis factor $\alpha$ (TNF $\alpha$ ), free fatty acids, and oxidative stress promote serine phosphorylation of IRS through activating stress-response kinases such as mitogen-activated protein kinase (MAPK) and c-Jun $\mathrm{N}$ terminal kinase (JNK), ultimately disrupting insulin signaling (Gual et al., 2005). In addition, chronic insulin stimulation downregulates insulin signaling via mammalian target of rapamycin complex (mTORC)-mediated serine/threonine phosphorylation of IRS which promotes the ubiquitination and degradation of IRS (Egawa et al., 2000; Pederson et al., 2001; Sun et al., 1999). We previously showed that the increase in IRS1 protein

elSSN: 0219-1032

(c) The Korean Society for Molecular and Cellular Biology. All rights reserved.

(C) This is an open-access article distributed under the terms of the Creative Commons Attribution-NonCommercial-ShareAlike 3.0 Unported License. To view a copy of this license, visit http://creativecommons.org/licenses/by-nc-sa/3.0/. 
level mediated by ginsenoid $\mathrm{Rg} 3$ increases insulin signaling and glucose uptake in L6 myotubes (Kim et al., 2009). Collectively, these results indicate that both IRS protein level and phosphorylation status regulate insulin signaling.

Retinoid X receptor ( $R X R)$ is a member of the nuclear receptor superfamily and regulates the transcription of target genes by forming a heterodimer with various nuclear receptors, including peroxisome proliferator-activated receptors (PPARs), liver $X$ receptors (LXRs), and thyroid hormone receptors (TRs) (Evans and Mangelsdorf, 2014; Heyman et al., 1992; Kliewer et al., 1992). RXR $\alpha$ expression is reported to be reduced in the high glucose state due to the increase in ROS (Singh et al., 2012). We previously demonstrated that impaired mitochondrial function caused by an mtDNA mutation reduced RXR $\alpha$ by ROSJNK pathway. Subsequent decrease in the interaction between PGC1 $\alpha$ and RXR $\alpha$ resulted in transcriptional repression of oxidative phosphorylation (OXPHOS) enzymes, aggravating mitochondrial dysfunction (Chae et al., 2013). Based on these results, we hypothesized that the reduction of $\mathrm{RXR} \alpha$ protein level in the setting of mitochondrial dysfunction is related to insulin signaling impairment. In this study, we investigated whether $\mathrm{RXR} \alpha$ protein levels are related to IRS1 expression and determined if $\mathrm{RXR} \alpha$ overexpression or activation can restore IRS1 expression and insulin signaling in the setting of mitochondrial dysfunction.

\section{MATERIALS AND METHODS}

Cell culture, adenovirus infection, and siRNA transfection C2C12 myoblasts were maintained in Dulbecco's minimum essential medium (DMEM) supplemented with $10 \%$ fetal bovine serum (FBS; Invitrogen, USA). Differentiation was induced by incubating with DMEM containing $2 \%$ horse serum (Invitrogen) for 4 days. COS7 cells were maintained in DMEM supplemented with $10 \%$ FBS. Myotubes were treated with 50 multiplicity of infection (MOI) Ad-GFP or Ad-RXR $\alpha$ for $24 \mathrm{~h}$ and then incubated with fresh media for $48 \mathrm{~h}$. Myotubes were treated with 9-cis retinoic acid (9cRA, Sigma, USA) or LG101506 (LG1506, Sigma). Myotubes were transfected with nonspecific siRNAs (siNS; Bioneer, Korea) or siRNAs of RXR $\alpha \quad(\operatorname{siRXR} \alpha$; Dharmacon, USA) using RNAiMAX (Invitrogen).

\section{Plasmids, adenovirus, and antibodies}

Three DNA fragments from $-1845 \mathrm{bp}$ to $-875 \mathrm{bp},-155 \mathrm{bp}$ to $875 \mathrm{bp}$, and $-998 \mathrm{bp}$ to $-875 \mathrm{bp}$ of the mouse IRS1 gene $(+1$ indicates translational start site) were inserted upstream of the luciferase gene in the pGL2-Basic (Promega, USA), and named IRS1(-1845)-Luc, IRS1 (-1155)-Luc and IRS1 (-998)Luc, respectively. Expression vectors were constructed by inserting the cDNAs of RXR $\alpha, \operatorname{PPAR} \alpha$, PPAR $\delta$ and PPAR $y$ into pcDNA 3.1 (Promega). To generate the adenovirus overexpressing human $R X R \alpha, R X R \alpha$ cDNA was inserted into the pAD-Track-CMV vector followed by recombination with a pAdEasy vector (Promega). Ad-RXR $\alpha$ was generated by transfecting the recombinant adenoviral DNA into human embryonic kidney (H293) cells. Antibodies against the proteins were used for western blot analyses and chromatin immunoprecipitation (ChIP): IRS1, pY612IRS1, RXR $\alpha$ PPAR $\delta$ and RNA Polymerase II (RNA pol II) (Santa Cruz Biotechnology, USA); AKT and pS473AKT (Cell Signaling, USA); and y-tubulin (Sigma).

\section{ATP measurement}

Cellular ATP levels were measured using ATPlite luminescence ATP detection assay system (PerkinElmer, USA). Briefly,
C2C12 myotubes were lysed in cell lysis solution and mixed with substrate solution before luminescence was measured.

\section{Western blot analysis}

C2C12 myotubes were lysed in $20 \mathrm{mM}$ Tris- $\mathrm{HCl}(\mathrm{pH} 7.4), 5 \mathrm{mM}$ $\mathrm{Na}_{4} \mathrm{P}_{2} \mathrm{O}_{7}, 100 \mathrm{nM} \mathrm{NaF}, 2 \mathrm{mM} \mathrm{Na} \mathrm{VO}_{4}$, and 1\% NP-40 buffer supplemented with protease inhibitors $(1 \mu \mathrm{g} / \mu \mathrm{l}$ aprotinin, $1 \mu \mathrm{g} / \mu \mathrm{l}$ leupeptin, and $1 \mathrm{mM}$ PMSF). About $20 \mu \mathrm{g}$ of proteins per lane were separated by SDS-PAGE and then transferred onto nitrocellulose membrane (Whatman, Germany). The bands were visualized by Enhanced Chemi-Luminescence (Pierce).

\section{RNA preparation and real-time $P C R$}

Total RNAs from the C2C12 myotubes were isolated by using TRIzol (Invitrogen). Real-time polymerase chain reaction (PCR) was performed using SYBR-master mix (Takara, Japan) and an ABI 7500 real-time PCR system (Applied Biosystems, USA). Each sample was analyzed in duplicate. The primers used for amplification were shown: for IRS1, 5' - GTT GGG CAG AAT AGG CCC TG -3' (forward) and 5'- AAT GCC TGT CCG CAT GTC AG -3' (reverse); for RXR $\alpha, 5^{\prime}$ - ACT GGT AGC CCC CAG CTC AA -3' (forward) and 5'- GAG CGG TCC CCA CAG ATA GC -3' (reverse); for glyceraldehyde 3-phosphate dehydrogenase (GAPDH), 5' - AGG TCG GTG TGA ACG GAT TTG -3' (forward) and 5'- TGT AGA CCA TGT AGT TGA GGT CA -3' (reverse).

Transient transfection and luciferase reporter assays COS7 cells were transfected with $0.3 \mu \mathrm{g}$ of reporter vectors and expression vectors using Lipofectamine with Plus Reagent (Invitrogen). An expression vector $(0.1 \mu \mathrm{g})$ for $\beta$-galactosidase was also transfected. pcDNA was added to transfect the same amount of DNA in each well. Cells were havested $24 \mathrm{~h}$ after transfection, and luciferase and $\beta$-galactosidase activities were measured.

\section{ChIP coupled with quantitative $P C R$}

C2C12 myotubes were infected with Ad-GFP or Ad-RXR $\alpha$ for 2 days and then treated with rotenone for an additional $24 \mathrm{~h}$. After cross-linking and DNA fragmentation, nuclear extracts were subjected to immunoprecipitation with antibodies against $\mathrm{RXR} \alpha$, PPAR $\delta$, RNA pol II, and control IgG. The primers used for amplification of the RXR $\alpha$ promoter region between -998 bp and 875 bp were: 5'- AAT GGT AGC GAG CAG GGA G -3' (forward) and 5' -CAC TCC AGA GGA GCA AAG CA- 3' (reverse).

\section{Statistical analysis}

Statistical analyses were performed using SPSS version 12.0 (SPSS Inc., USA). Significant differences were assessed using Mann-Whitney $U$ tests. A $P$ values below 0.05 were considered statistically significant.

\section{RESULTS}

IRS1 and RXR $\alpha$ protein levels are reduced by OXPHOS complex inhibitors

We first determined whether IRS1 and RXR $\alpha$ protein levels were affected by mitochondrial dysfunction. C2C2 myoblasts were differentiated to myotubes, and then treated with the OXPHOS complex inhibitors rotenone (complex I inhibitor) and antimycin A (complex III inhibitor) for $24 \mathrm{~h}$. Both IRS1 and $\mathrm{RXR} \alpha$ protein levels were markedly decreased after treatments (Fig. 1A). Real time quantitative PCR was used to determine whether the reduction of IRS1 and RXR $\alpha$ proteins was due to 

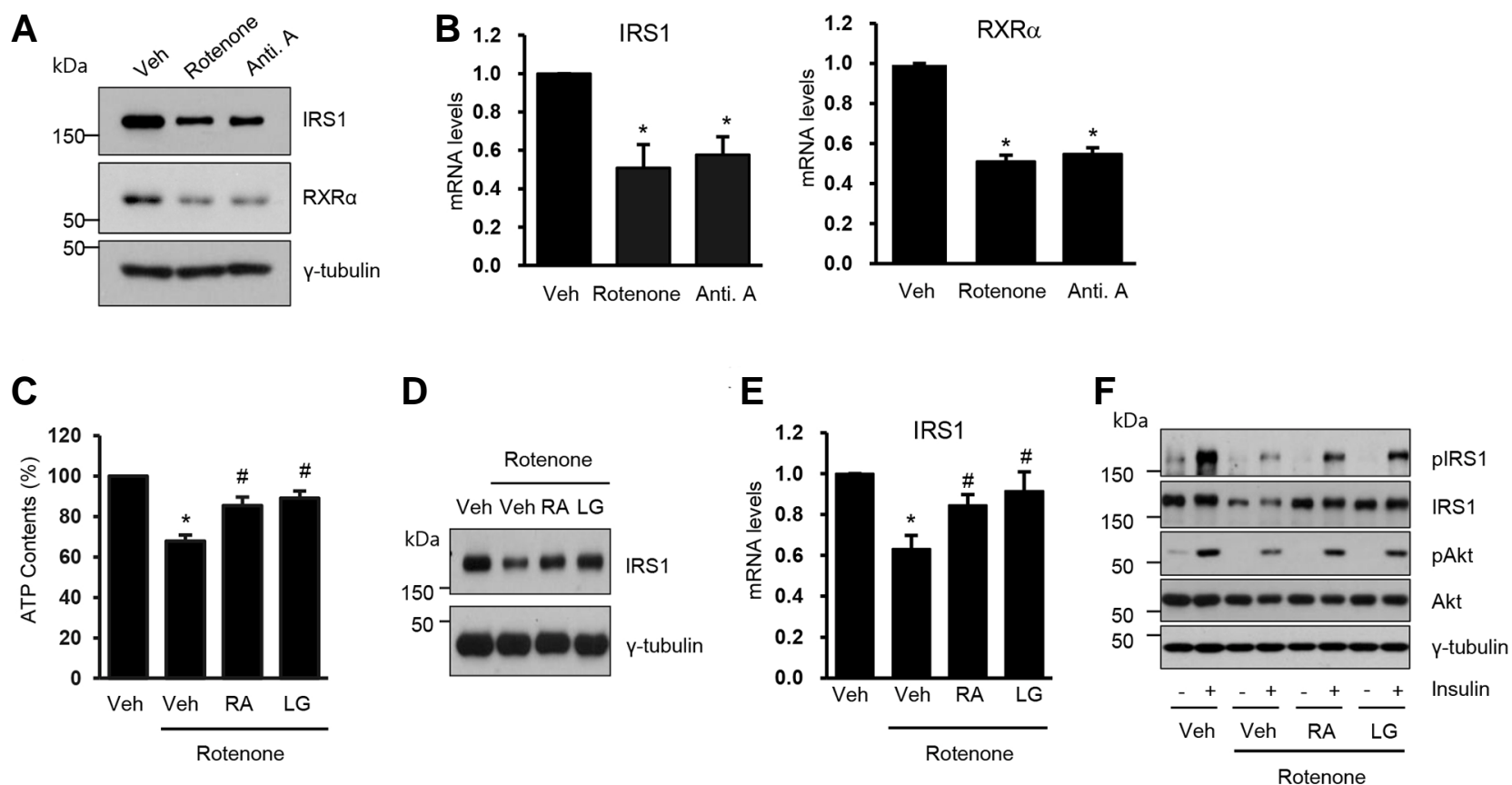

Fig. 1. RXR $\alpha$ agonists restore mitochondrial function and IRS transcription impaired by rotenone. $\mathrm{C} 2 \mathrm{C} 12$ myoblasts were differentiated to myotubes and treated with rotenone $(3 \mu \mathrm{M})$ or antimycin $A(10 \mu \mathrm{M})$ for $24 \mathrm{~h}$. (A) Cells were lysed and then Western blot analyses were performed with the specific antibodies. (B) Total RNAs were isolated and real time quantitative PCR was performed. The mRNA levels of each gene were normalized by that of GAPDH. The value of Veh was set to 1 and the others were expressed as the relatives to that. The data are the means \pm SEM of 3 experiments. * $P<0.05$ vs. Veh. (C-F) C2C12 myotubes were treated with rotenone $(3 \mu \mathrm{M})$ for $24 \mathrm{~h}$ and then the media was replaced to the fresh media containing DMSO (Veh), 9cRA $(5 \mu \mathrm{M})$ or LG1506 $(2 \mu \mathrm{M})$ for $18 \mathrm{~h}$. (C) Cells were lysed and ATP contents were measured $(\mathrm{n}=6)$. ${ }^{*}, P<0.05$ vs. Veh; \#, $P<0.05$ vs. rotenone only. (D) Cell lysates were subjected to Western blot analysis. (E) The IRS1 mRNA levels were measured by real time PCR $(n=5)$. ${ }^{*}, P<0.05$ vs. Veh; \#, $P<0.05$ vs. rotenone only. (F) Insulin (100 nM) was added $15 \mathrm{~min}$ before harvesting. Cell lysates were subjected to the Western blot analysis.

decreased mRNA levels. Both IRS1 and RXR $\alpha$ mRNA levels were significantly reduced following treatment with rotenone or antimycin A (Fig. 1B), suggesting that IRS1 and RXR $\alpha$ transcription are suppressed in the setting of mitochondrial dysfunction.

\section{$R X R \alpha$ agonists reverse the effects of rotenone on IRS1} expression and insulin signaling

Because we previously showed that activation of $\mathrm{RXR} \alpha$ by its agonists significantly recovered mitochondrial function in cybrid cells having mutant mitochondrial DNA (Chae et al., 2013), we tested whether RXR $\alpha$ agonist treatment enhances IRS1 expression and restores insulin signaling impaired by mitochondrial dysfunction. $\mathrm{C} 2 \mathrm{C} 12$ myotubes were treated with rotenone for $24 \mathrm{~h}$ and then treated with two different types of agonists in fresh media for $18 \mathrm{~h}$ : 9-cis-retinoic acid (9cRA) and LG101506 (LG1506). ATP levels were increased after the cells were incubated with 9cRA or LG1506, indicating that mitochondria function was partially recovered following treatment with an $\mathrm{RXR} \alpha$ agonist (Fig. 1C). IRS1 protein levels, which had been reduced by rotenone treatment, were restored to baseline when 9cRA or LG1506 was added (Fig. 1D). A similar effect was observed with IRS1 mRNA levels (Fig. 1E). In addition to the recovery of IRS1 expression levels, rotenone-induced insulin signaling impairment was significantly alleviated by the addition of either $\mathrm{RXR} \alpha$ agonist (Fig. 1F). These results suggest that $\mathrm{RXR} \alpha$ activation recovers rotenone-mediated decreases in IRS1 transcription and ATP production and that the quantitative recovery of IRS1 protein is important for normal insulin signaling.

$R X R \alpha$ overexpression restores IRS1 transcription in the presence of rotenone

Next, we tested whether RXR $\alpha$ protein level itself affects rotenone-suppressed IRS1 mRNA or protein levels. When RXR $\alpha$ was overexpressed using an adenoviral system, IRS1 protein levels were also increased in the presence of rotenone (Fig 2A). Consistent with this finding, IRS1 mRNA levels were also increased following $\mathrm{RXR} \alpha$ overexpression (Fig. $2 \mathrm{~B}$ ). In addition, $\mathrm{RXR} \alpha$ overexpression prevented rotenone-induced insulin signaling impairment (Fig. 2C). We also assessed the effect of $\mathrm{RXR} \alpha$ overexpression on mitochondrial function. ATP contents were significantly recovered by $\mathrm{RXR} \alpha$ overexpression in the presence of rotenone (Fig. 2D). These results suggest that $\mathrm{RXR} \alpha$ overexpression was sufficient to reverse decreased IRS transcription and mitochondrial dysfunction induced by rotenone.

\section{RXRo directly regulates IRS1 transcription}

Although our data clearly showed that $R X R \alpha$ activation or overexpression ameliorated mitochondrial dysfunction and increased IRS1 transcription, it was not clear whether RXR $\alpha$ directly regulates IRS1 transcription or if the recovery of IRS1 transcription is a secondary effect of improved mitochondrial function. We therefore tested whether RXR $\alpha$ affected IRS1 transcription in the myotubes that were not treated with rotenone. RXR $\alpha$ overexpression increased IRS1 mRNA and pro- 

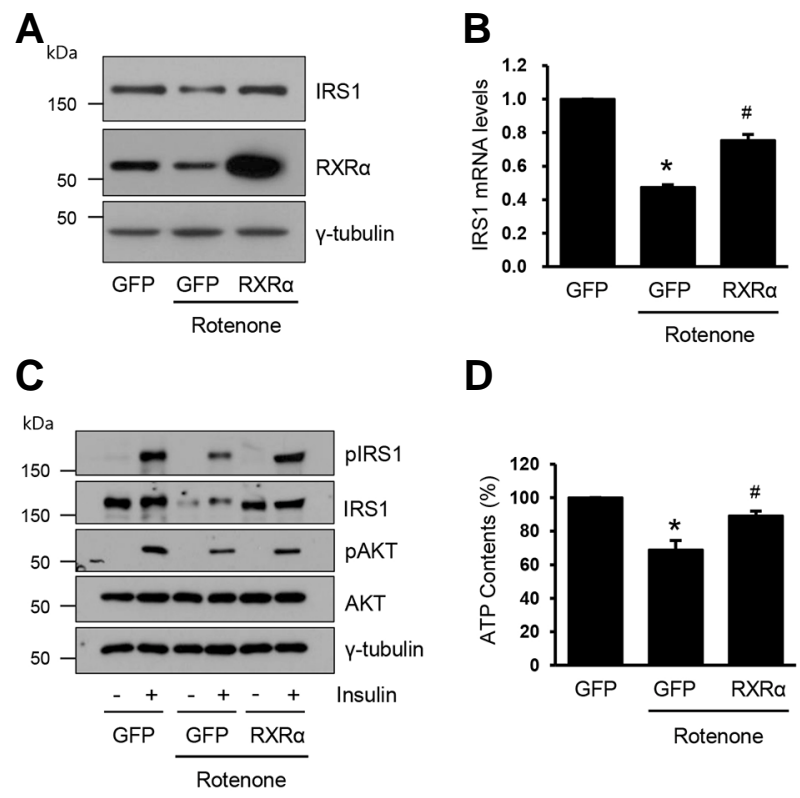

D

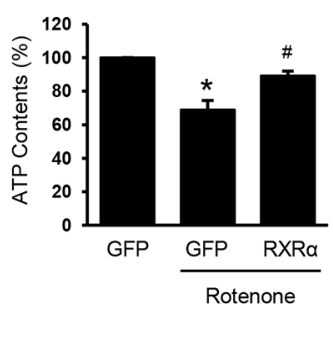

Fig. 2. RXR $\alpha$ overexpression prevents rotenone-induced suppression of IRS1 transcription. C2C12 myotubes were infected with Ad$\mathrm{RXR} \alpha(50 \mathrm{MOI})$ for $48 \mathrm{~h}$ and then treated with rotenone for an additional $24 \mathrm{~h}$. (A) Western blot analysis and (B) real-time PCR were performed $(n=6)$. $P<0.05$ vs. Ad-GFP; \#, $P<0.05$ vs. Ad-GFP with rotenone. (C) Insulin was treated for 15 min before harvesting and western blot analysis was performed. (D) Cellular ATP contents were measured $(\mathrm{n}=7)$. ${ }^{*}, P<0.05$ vs. Ad-GFP; $\#, P<0.05$ vs. AdGFP with rotenone.

tein levels in a dose-dependent manner (Figs. 3A and 3B). Similarly, activation of RXR $\alpha$ by LG1506 treatment also increased IRS1 mRNA and protein levels. However, the RXR $\alpha$ agonist 9cRA did not increase IRS1 mRNA or protein levels (Figs. 3C and 3D). We also examined the effect of $R X R \alpha$ knockdown on IRS1 mRNA levels and found that they were significantly reduced when siRNAs against $R X R \alpha$ were transfected into $\mathrm{C} 2 \mathrm{C} 12$ myotubes (Fig. 3E). These results suggest that $R X R \alpha$ is directly involved in IRS1 transcription independently of mitochondrial function, even though 9cRA did not increase IRS1 mRNA levels.

$R X R \alpha$ binds to the IRS1 promoter and augments its activity To confirm that $\mathrm{RXR} \alpha$ directly binds to the IRS1 promoter and activates the transcription of IRS1, DNA fragments containing three different lengths of 5 '-flanking region of the mouse IRS1 gene (from $-1845,-1155$, or -998 bp to $-875 \mathrm{bp}$ ) were linked to the luciferase reporter gene and then transfected into COS7 cells with an $R X R \alpha$ expression vector (Fig. 4A). RXR $\alpha$ increased and LG1506 further increased the luciferase activities of the (-1845)-Luc and (-1155)-Luc constructs but not the (-998)-Luc construct (Fig. 4B). This finding suggests that a cisacting element(s) for $R X R \alpha$ is located between -1155 bp and $998 \mathrm{bp}$ in the IRS1 promoter. In contrast, 9cRA did not increase the luciferase activity further (data not shown). Next, we identified the partner of $\mathrm{RXR} \alpha$ for binding to the IRS1 promoter region. Based on a report that LG1506 is a specific activator for the RXR $\alpha / P P A R$ heterodimer, we tested whether PPAR $\alpha$, PPAR $\delta$, and PPARy, could increase the luciferase activity of
A

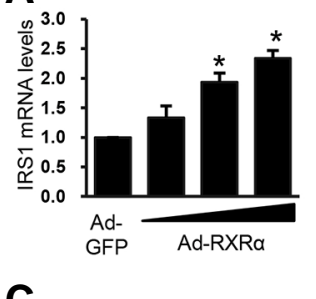

C

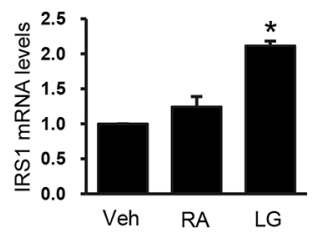

D

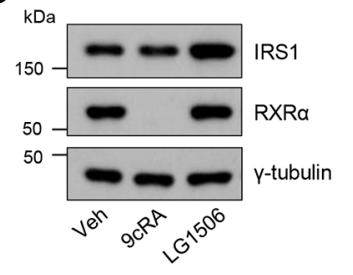

$\boldsymbol{E}$
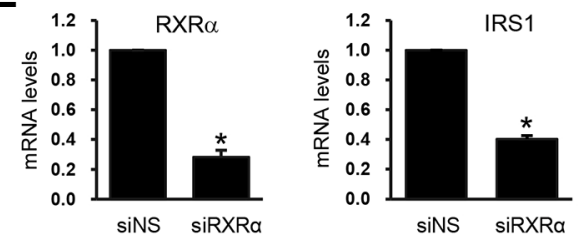

Fig. 3. $\mathrm{RXR} \alpha$ directly regulates IRS1 transcription. (A, B) $\mathrm{C} 2 \mathrm{C} 12$ myotubes were infected with $A d-R X R \alpha$ in different dosages (10, 30 and $50 \mathrm{MOI}$ ) for $48 \mathrm{~h}$. Cell lysates were subjected to real time PCR (A) and western blot analysis (B). The mRNA levels of control cells (Ad-GFP infected) was set to 1 and the other values were expressed as the relatives to that $(\mathrm{n}=4)$. ${ }^{*}, P<0.05$ vs. Ad-GFP infected cells. (C, D) C2C12 myotubes were treated with 9cRA (5 $\mu \mathrm{M})$ or LG1506 $(2 \mu \mathrm{M})$ for $24 \mathrm{~h}$, and then real time PCR $(\mathrm{C})$ and western blot analysis (D) were performed $(n=5)$. ${ }^{*}, P<0.05$ vs. Veh. (E) C2C12 myotubes were transfected with siNS or siRXR (50 $\mathrm{nM})$. Total RNAs were prepared 3 days after siRNA transfection and then real time PCR was performed $(n=3) .{ }^{*}, P<0.05$ vs. siNS.

the IRS1 (-1155)-Luc construct. Co-transfection of PPAR expression vectors further increased luciferase activity, but PPAR $\alpha$ and PPARy vectors did not (Fig. 4C), indicating that the $\mathrm{RXR} \alpha / \mathrm{PPAR} \delta$ heterodimer binds to the promoter region of the IRS1 gene. To confirm that RXR $\alpha$ and PPAR $\delta$ directly bind to the region between -1155 bp and -998 bp, ChIP was performed with RXR $\alpha$, PPAR $\delta$, or RNA Pol II antibodies in $\mathrm{C} 2 \mathrm{C} 12$ myotubes, and real-time PCR was performed using primers flanking the region (from -1155 bp to $-998 \mathrm{bp}$ ). While bindings of RXR $\alpha$, PPAR $\delta$, and RNA Pol II were decreased following rotenone treatment, bindings of these factors to the region were significantly increased when $\mathrm{RXR} \alpha$ was overexpressed. These results indicated that IRS1 transcription was augmented by $\mathrm{RXR} \alpha$ overexpression (Fig. 4D). Collectively, RXR $\alpha$ forms a heterodimer with PPAR $\delta$ that directly binds to the IRS1 promoter region between $-1155 \mathrm{bp}$ and $-998 \mathrm{bp}$ and regulates IRS1 transcription level.

\section{DISCUSSION}

We found that IRS1 and RXR $\alpha$ protein levels were dramatically reduced when mitochondrial dysfunction was induced by OXPHOS complex inhibitors in myotubes. Based on this finding, 
A

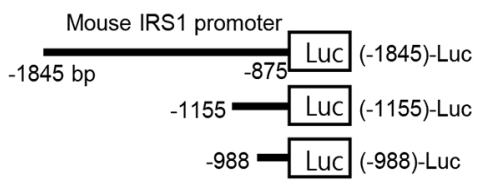

C

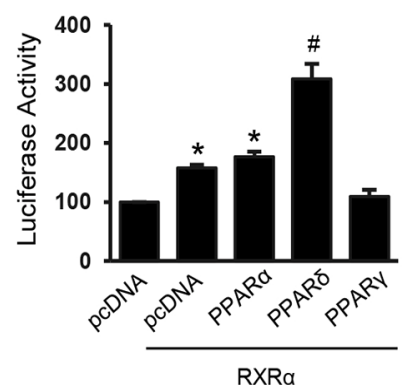

$B$

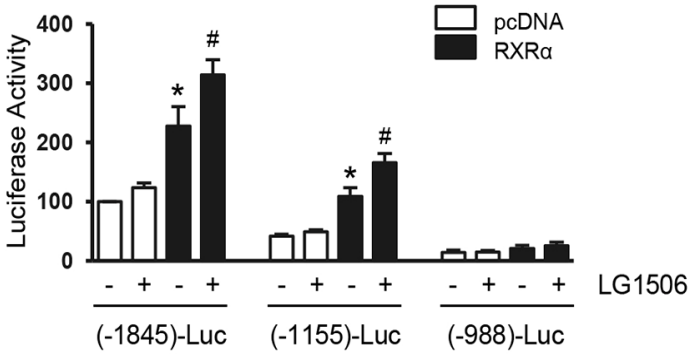

D

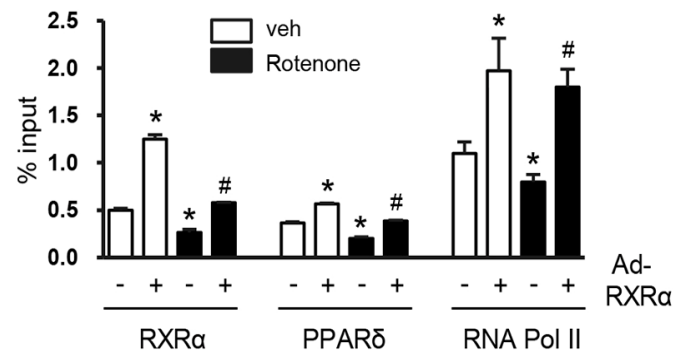

Fig. 4. $\mathrm{RXR} \alpha$ directly binds to the IRS1 promoter. (A) A diagram of three different lengths of IRS1 promoter-luciferase constructs, IRS1 (-1845)-Luc, IRS1 (-1155)-Luc and IRS1 (-998)-Luc. (B) COS7 cells were transfected with one of the IRS1 promoter-Luc constructs and RXR $\alpha$ expression vector (pcDNA$R X R \alpha)$. Three hours after the transfection, cells were treated with LG1506 $(2 \mu \mathrm{M})$ for an additional $18 \mathrm{~h}$. Luciferase activity of the cells transfected with IRS1 $(-1845)$-Luc was set to 100 and the other values were expressed as the relatives to that $(\mathrm{n}=5)$. ${ }^{*}, P$ $<0.05$ vs pcDNA without LG1506 for each construct. \#, $P<0.05$ vs. pcDNA-RXR $\alpha$ without LG1506. (C) COS7 cells were transfected with IRS1 (-1155) Luc, expression vectors for RXR $\alpha$, PPAR $\alpha$, PPAR $\delta$ or PPARy. Luci ferase activity of the cells transfected with IRS1

(-1155)-Luc without RXR $\alpha$ or PPAR expression vectors was set to 100 and the others were expressed as the relatives to that $(n=4)$. *, $P<$ 0.05 vs. cells transfected with IRS1 (-1155)-Luc only. \#, $P<0.05$ vs. cells transfected with IRS1 (-1155)-Luc and pcDNA-RXR $\alpha$ (D) C2C12 myotubes were infected with Ad-RXR $\alpha(50 \mathrm{MOI})$ for $48 \mathrm{~h}$, and then treated with rotenone for an additional $24 \mathrm{~h}$. Cells were subjected to ChIP. Data are the means \pm SEM $(\mathrm{n}=3)$. ${ }^{*}, P<0.05$ vs. Ad-GFP infected cells not treated with rotenone. \#, $P<0.05$ vs. Ad-GFP infected cells treated with rotenone.

we investigated the relationship between IRS1 and $R X R \alpha$ expression. We showed that $\mathrm{RXR} \alpha$ overexpression or activation by its agonists accelerated the recovery of mitochondrial function and also increased IRS1 transcription, which in turn activated the insulin signaling pathway. Our data also indicate that $\mathrm{RXR} \alpha$ binds to the IRS1 promoter and thus directly affects its transcription. LG1506 is reported as a heterodimer-selective modulator of $R X R \alpha$ : it activates $R X R \alpha$ : PPAR but not $R X R \alpha$ : LXR $\alpha$ or RXR $\alpha$ : FXR $\alpha$ heterodimers (Leibowitz et al., 2006). Consistently, our data showed that PPAR $\delta$ bound to the IRS1 promoter, suggesting that $R X R \alpha$ : PPAR $\delta$ heterodimers are involved in IRS1 transcriptional regulation.

Unlike LG1506, treatment of 9cRA increased IRS1 transcription when mitochondria were damaged but did not affect IRS1 transcription under control conditions. At this time, we cannot justify why 9cRA did not increase IRS1 transcription levels. We observed that $\mathrm{RXR} \alpha$ protein levels were dramatically reduced by the addition of 9cRA but not LG1506 (Fig. 3D). Liganddependent ubiquitination of $R X R \alpha$ has been reported by several researchers: conformational changes induced by binding of specific ligands such as RA or LGD1268 are recognized by ubiquitination enzymes, which leads to proteasome-mediated degradation of $\mathrm{RXR} \alpha$ (Gianni et al., 2003; Osburn et al., 2001). In contrast, binding of other types of ligand such as LG1506 may not induce the conformational change preferential for ubiquitination. The difference may explain the differential regulation of IRS1 transcription by 9cRA and LG1506. Based on our study, we postulate that $R X R \alpha$ increases IRS1 transcription by two different mechanisms: directly binding to the IRS1 promoter and indirectly recovering mitochondrial dysfunction. However, further studies are needed to determine how mitochondrial function is related to IRS1 transcription.
Although we confirmed that $\mathrm{RXR} \alpha$ bound to the promoter region between -1155 bp and 998 bp of the IRS1 gene, the precise binding site remains to be identified. It is well known that RXR:PPAR heterodimers bind to the direct repeat motif-1 (DR1); however, a well conserved DR1 site was not found in the region, indicating that they may bind to the region with quite low affinity. This could explain the findings that $R X R \alpha$ abundance was important for the binding and that 9cRA did not further increase IRS1 promoter activity (data not shown).

Our results showed that IRS1 mRNA and protein levels were dramatically reduced following treatment with OXPHOS inhibitors. A previous report showed that the induction of specific miRNAs is involved in suppressing IRS1 expression in the setting of mitochondrial dysfunction (Ryu et al., 2011). Regarding the involvement of RXR $\alpha$ in the regulation of IRS1 transcription in our study, reduced $R X R \alpha$ levels are likely partially involved in the suppression of IRS1 transcription in cells with impaired mitochondria. In addition to mitochondrial dysfunction, reduced IRS1 protein levels are often observed in the muscles of insulinresistant animal models and myotubes (Macotela et al., 2011; Yuzefovych et al., 2012). These findings suggest that the reduction of IRS1 protein levels is a typical phenomenon of insulin resistance, however, little is known about IRS1 transcriptional regulation. Our data provide evidence that IRS1 transcription is regulated by $R X R \alpha$. There are several reports showing in vivo study of RXR $\alpha$ agonists: LG100268 improves glycemic control and decreases cardiovascular risk in diabetic mice, and LG100754, a selective RXR:PPAR $\gamma$ agonist, decreases glucose levels of $d b / d b$ mice (Cesario et al., 2001; Lenhard et al., 1999). Because, according to our study, LG1506 has beneficial effects on insulin resistance in myotubes, it will be worth to do in vivo study for clinical application. 


\section{ACKNOWLEDGMENTS}

This study was supported by the National Research Foundation of Korea (NRF) grant funded by the Korean government (Ministry of Education, Science and Technology; NRF-20062005410).

\section{REFERENCES}

Anderson, E.J., Lustig, M.E., Boyle, K.E., Woodlief, T.L., Kane, D.A., Lin, C.T., Price, J.W., 3rd, Kang, L., Rabinovitch, P.S., Szeto, H.H., et al. (2009). Mitochondrial H2O2 emission and cellular redox state link excess fat intake to insulin resistance in both rodents and humans. J. Clin. Invest. 119, 573-581.

Cesario, R.M., Klausing, K., Razzaghi, H., Crombie, D., Rungta, D., Heyman, R.A., and Lala, D.S. (2001). The rexinoid LG100754 is a novel RXR:PPARgamma agonist and decreases glucose levels in vivo. Mol. Endocrinol. 15, 1360-1369.

Chae, S., Ahn, B.Y., Byun, K., Cho, Y.M., Yu, M.H., Lee, B., Hwang, D., and Park, K.S. (2013). A systems approach for decoding mitochondrial retrograde signaling pathways. Sci. Signal. 6, rs4.

Egawa, K., Nakashima, N., Sharma, P.M., Maegawa, H., Nagai, Y. Kashiwagi, A., Kikkawa, R., and Olefsky, J.M. (2000). Persistent activation of phosphatidylinositol 3-kinase causes insulin resistance due to accelerated insulin-induced insulin receptor substrate-1 degradation in 3T3-L1 adipocytes. Endocrinology 141, 19301935.

Evans, R.M., and Mangelsdorf, D.J. (2014). Nuclear receptors, RXR, and the big bang. Cell 157, 255-266.

Fisher-Wellman, K.H., and Neufer, P.D. (2012). Linking mitohondrial bioenergetics to insulin resistance via redox biology. Trends Endocrinol. Metab. 23, 142-153.

Gianni, M., Tarrade, A., Nigro, E.A., Garattini, E., and Rochette-Egly, C. (2003). The AF-1 and AF-2 domains of RAR gamma 2 and RXR alpha cooperate for triggering the transactivation and the degradation of RAR gamma $2 / R X R$ alpha heterodimers. J. Biol. Chem. 278, 34458-34466.

Gual, P., Le Marchand-Brustel, Y., and Tanti, J.F. (2005). Positive and negative regulation of insulin signaling through IRS-1 phosphorylation. Biochimie 87, 99-109.

Heyman, R.A., Mangelsdorf, D.J., Dyck, J.A., Stein, R.B., Eichele, G., Evans, R.M., and Thaller, C. (1992). 9-cis retinoic acid is a high affinity ligand for the retinoid $X$ receptor. Cell 68, 397-406.

Kelley, D.E., He, J., Menshikova, E.V., and Ritov, V.B. (2002). Dysfunction of mitochondria in human skeletal muscle in type 2 diabetes. Diabetes 51, 2944-2950.

Kelley, D.E., and Simoneau, J.A. (1994). Impaired free fatty acid utilization by skeletal muscle in non-insulin-dependent diabetes mellitus. J. Clin. Invest. 94, 2349-2356.

Kim, M., Ahn, B.Y., Lee, J.S., Chung, S.S., Lim, S., Park, S.G., Jung, H.S., Lee, H.K., and Park, K.S. (2009). The ginsenoside $\mathrm{Rg} 3$ has a stimulatory effect on insulin signaling in L6 myotubes. Biochem. Biophys. Res. Commun. 389, 70-73.

Kliewer, S.A., Umesono, K., Noonan, D.J., Heyman, R.A., and Evans, R.M. (1992). Convergence of 9-cis retinoic acid and peroxisome proliferator signalling pathways through heterodimer formation of their receptors. Nature 358, 771-774.
Leibowitz, M.D., Ardecky, R.J., Boehm, M.F., Broderick, C.L., Caragna, M.A., Crombie, D.L., D’Arrigo, J., Etgen, G.J., Faul, M.M., Grese, T.A., et al. (2006). Biological characterization of a heterodimer-selective retinoid $X$ receptor modulator: potential benefits for the treatment of type 2 diabetes. Endocrinology 147, 1044-1053.

Lenhard, J.M., Lancaster, M.E., Paulik, M.A., Weiel, J.E., Binz, J.G., Sundseth, S.S., Gaskill, B.A., Lightfoot, R.M., and Brown, H.R (1999). The RXR agonist LG100268 causes hepatomegaly, improves glycaemic control and decreases cardiovascular risk and cachexia in diabetic mice suffering from pancreatic beta-cell dysfunction. Diabetologia 42, 545-554.

Lowell, B.B., and Shulman, G.I. (2005). Mitochondrial dysfunction and type 2 diabetes. Science 307, 384-387.

Macotela, Y., Emanuelli, B., Bang, A.M., Espinoza, D.O., Boucher, J., Beebe, K., Gall, W., and Kahn, C.R. (2011). Dietary leucine-an environmental modifier of insulin resistance acting on multiple levels of metabolism. PLoS One 6, e21187.

Martin, S.D., and McGee, S.L. (2014). The role of mitochondria in the aetiology of insulin resistance and type 2 diabetes. Biochim. Biophys. Acta 1840, 1303-1312.

Morino, K., Petersen, K.F., Dufour, S., Befroy, D., Frattini, J., Shatzkes, N., Neschen, S., White, M.F., Bilz, S., Sono, S., et al. (2005). Reduced mitochondrial density and increased IRS-1 serine phosphorylation in muscle of insulin-resistant offspring of type 2 diabetic parents. J. Clin. Invest. 115, 3587-3593.

Osburn, D.L., Shao, G., Seidel, H.M., and Schulman, I.G. (2001). Ligand-dependent degradation of retinoid $\mathrm{X}$ receptors does not require transcriptional activity or coactivator interactions. Mol. Cell. Biol. 21, 4909-4918.

Pederson, T.M., Kramer, D.L., and Rondinone, C.M. (2001). Serine/ threonine phosphorylation of IRS-1 triggers its degradation: possible regulation by tyrosine phosphorylation. Diabetes 50 , 24-31.

Ritov, V.B., Menshikova, E.V., He, J., Ferrell, R.E., Goodpaster, B.H., and Kelley, D.E. (2005). Deficiency of subsarcolemmal mitochondria in obesity and type 2 diabetes. Diabetes 54, 8-14.

Ryu, H.S., Park, S.Y., Ma, D., Zhang, J., and Lee, W. (2011). The induction of microRNA targeting IRS-1 is involved in the development of insulin resistance under conditions of mitochondrial dysfunction in hepatocytes. PLoS One 6, e17343.

Simoneau, J.A., and Kelley, D.E. (1997). Altered glycolytic and oxidative capacities of skeletal muscle contribute to insulin resistance in NIDDM. J. Appl. Physiol. 83, 166-171.

Singh, A.B., Guleria, R.S., Nizamutdinova, I.T., Baker, K.M., and Pan, J. (2012). High glucose-induced repression of RAR/RXR in cardiomyocytes is mediated through oxidative stress/JNK signaling. J. Cell. Physiol. 227, 2632-2644.

Sun, X.J., Goldberg, J.L., Qiao, L.Y., and Mitchell, J.J. (1999). Insulin-induced insulin receptor substrate-1 degradation is mediated by the proteasome degradation pathway. Diabetes 48 , 1359-1364.

Yuzefovych, L.V., Solodushko, V.A., Wilson, G.L., and Rachek, L.I. (2012). Protection from palmitate-induced mitochondrial DNA damage prevents from mitochondrial oxidative stress, mitochondrial dysfunction, apoptosis, and impaired insulin signaling in rat L6 skeletal muscle cells. Endocrinology 153, 92-100. 